\title{
RESEARCH
}

Open Access

\section{Test-retest reliability of nerve and muscle morphometric characteristics utilizing ultrasound imaging in individuals with unilateral sciatica and controls}

Hadi Sarafraz ${ }^{1,2}$, Mohammad Reza Hadian ${ }^{3 *}$, Niloofar Ayoobi Yazdi ${ }^{4}$, Gholamreza Olyaei ${ }^{5}$, Hossein Bagheri ${ }^{5}$, Shohreh Jalaie ${ }^{6}$ and Omid Rasouli ${ }^{7}$

\begin{abstract}
Background: Ultrasound imaging has been suggested for studying the structure and function of nerves and muscles; however, reliability studies are limited to support the usage. The main aim of this study was to explore the intrarater within-session reliability of evaluating the sciatic nerve and some related muscles morphology by ultrasound imaging.

Methods: Three B-mode images from two scans (transverse and longitudinal) were acquired from the multifidus, biceps femoris, soleus and medial gastrocnemius muscles bilaterally from 15 participants with sciatica and 15 controls in one session, 1-h apart. The data were collected from March to July 2017. Contraction ratio was measured only by longitudinal scan, while the echo intensity was measured using maximum rectangular region of interest in two scans (transverse and longitudinal) for all muscles. Cross-sectional area, direct (tracing) and indirect (ellipsoid formula) methods were used to measure the sciatic nerve. Intraclass correlation coefficient (ICC 3,1), standard error of measurement and minimal detectable change were calculated.

Results: Good to high ICCs (0.80-0.96) were found for muscle contraction ratio in the longitudinal scans in all the muscles in both sciatica and control groups. For echo intensity measurements ICCs ranged from moderate to high, with higher ICCS seen with the maximum region of interest in the transverse scans. The minimal detectable change values ranged between 0.11 and $0.53 \mathrm{~cm}$ for contraction ratio.

Conclusions: Ultrasound imaging has high intrarater within-session reliability for assessing the sciatic nerve Crosssectional area and muscle contraction ratios. Transverse scans with the maximum region of interest result in higher reliability. The sciatic Cross-sectional area is most accurately measured utilizing the direct tracing method rather than the indirect ellipsoid method.
\end{abstract}

Keywords: Reproducibility, Ultrasonography, Skeletal muscle, Low back pain, Sciatic nerve

\footnotetext{
* Correspondence: hadianrs@sina.tums.ac.ir

${ }^{3}$ Department of Physiotherapy, School of Rehabilitation, Tehran University of

Medical Sciences, International Campus (TUMS, IC-TUMS), Brain and Spinal

Injury Research Center (BASIR), Tehran, Iran

Full list of author information is available at the end of the article
}

(c) The Author(s). 2018 Open Access This article is distributed under the terms of the Creative Commons Attribution 4.0 International License (http://creativecommons.org/licenses/by/4.0/), which permits unrestricted use, distribution, and reproduction in any medium, provided you give appropriate credit to the original author(s) and the source, provide a link to the Creative Commons license, and indicate if changes were made. The Creative Commons Public Domain Dedication waiver (http://creativecommons.org/publicdomain/zero/1.0/) applies to the data made available in this article, unless otherwise stated. 


\section{Background}

Low back pain with a clear pathoanatomical diagnosis of lumbar radiculopathy is a common type of specific low back pain (LBP) [1]. Sciatic neuropathy due to disc herniation is the most common peripheral entrapment neuropathy that seen in musculoskeletal setting [2]. Sciatica due to disc herniation compressing a nerve root may lead to disturbance in contractibility of multifidus, biceps femoris, and gastrosoleus muscles innervated by this nerve. To investigate the nerve and muscle morphometric characteristics in sciatica, neuromuscular ultrasound imaging has been suggested for the assessment of both nerve and muscle in patients with entrapment neuropathy [3]. Compared to the magnetic resonance imaging, ultrasound imaging is less expensive, more accessible, feasible and cost-effective [4]. This technique can provide useful information about the muscle function / dysfunction [5]. However, regarding the muscle size, it is important to ensure that the subject is cooperating for either full relaxation or contraction because the muscle dimensions change during contraction / relaxation. Intrarater reliability determines the stability of data recorded by one individual across two or more trials. Having acceptable reliability is essential for any kind of measurement and for making valid decisions [6].

In addition to muscle contraction ratio (i.e. contracted thickness/rest thickness), muscle echo intensity has been recently suggested as a potential marker of muscle-tissue status that can affect the muscle function [7]. Normal muscle displays as a moderately hypoechoic structure in the B-mode ultrasound image because of the low reflection of the ultrasound wave (low echo intensity). Muscles have a speckled look in a transverse scan, due to higher echo intensity of the perimysium around muscle fiber bundles relative to the proper muscle tissue. Longitudinal scans usually have better contrast in echo intensity between the muscle fascicles and the perimysium connective tissue, and this contrast in echo intensity is useful for defining the muscle boundaries and characterization of the muscle architecture $[5,8]$. The echo intensity in an ultrasound image can be determined as the average intensity of the pixels inside the target muscle by a scale of gray levels within a given region of interest (ROI) [9]. In the literature, the reliability of echo intensity measures for muscles is controversial and there are still questions regarding the most appropriate method to collect such measures. In addition, the appropriate size of ROI is questionable and some authors such as Caresio et al. (2015) have suggested including as much of a muscle as possible, but avoiding surrounding fascia and bones [7]. Imaging whole section of a muscle may be important since internal fascia and nonhomogeneous distribution of echo intensity might affect the measures. The orientation of muscle bundles might also affect the reliability of echo intensity measures, particularly in longitudinal scans [7].

Sciatica may cause swelling of the sciatic nerve at the posterior thigh level. Increased size and loss of echogenicity of compressed nerves are not well understood. This may partly result from increased vascularity and edema around the nerve. Some studies have investigated the reliability of nerve size and echogenicity of peripheral nerves [10-13]. However, little is known about the reliability of the cross-sectional area (CSA) and echogenicity of the sciatic nerve at the posterior mid-thigh. Moreover, the reliability of measuring nerve enlargement by ultrasound has received little attention in the literature, and there is limited information on the reliability of morphometric characteristics (CSA and echo intensity) of the sciatic nerve, e.g. in patients with sciatica due to disc herniation. One study has investigated the reliability and validity of ultrasound imaging on the sciatic nerve CSA and muscle thickness in healthy subjects with small sample size [14]; however, there is no study conducted to determine the reliability of the sciatic nerve CSA and echo intensity by ultrasound imaging in sciatica patients [15].

Therefore, the main aim of this study was to investigate the intrarater within-day reliability of the nerve and muscle morphometric characteristics in both transverse and longitudinal scans, while utilizing a max rectangular ROI or entire scanned section of the muscle (max ROI) in sciatica patients and controls.

\section{Methods \\ Participants}

Fifteen patients, aged 30-50 years with the complaint of LBP with unilateral radiculopathy (sciatica) lasting for a minimum of three consecutive months participated voluntarily in this study (Table 1). Diagnosis was made by a neurosurgeon based on the criteria recommended by Nijs et al. (2015) (LBP with at least one symptom of pain, numbness, or tingling radiating down to the leg and / or foot)

Table 1 Demographic data (mean \pm SD) of the participants in each group (sciatica and control)

\begin{tabular}{lllllll}
\hline Group & Age (year) & BMI $(\mathrm{kg} / \mathrm{m} 2)$ & Tenger scale & NPRS $^{\mathrm{b}}$ back & NPRS Leg & ODI $^{\mathrm{c}}$ Range \\
\hline Sciatica $(n=15)$ & $42 \pm 14.1$ & $24 \pm 2.3$ & $3 \pm 0.1$ & $4.8 \pm 1.9$ & $5.8 \pm 1.6$ & $35.7-40.1 \pm 11.9-13.7$ \\
Control $(n=15)$ & $41 \pm 13.9$ & $23.6 \pm 2$ & $3 \pm 0.1$ & - & - & - \\
\hline
\end{tabular}

BMI Body mass index; ${ }^{\mathrm{a} T e g n e r}$ Activity Scale, score $0-10$ where 0 represents sick leave or disability pension, and 10 is participation in competitive sports. ${ }^{\mathrm{b}}$ Numeric Pain Rating Scale, on scale 0-10, a score 1-3 indicates mild pain, 4-6 indicates moderate pain, 7-10 indicates severe pain. ${ }^{\mathrm{C}} \mathrm{ODI}$ : Oswestry Disability Index, on a percentage scale, scores from 0 to $20 \%$ indicate a minimal disability, $21-40 \%$ indicate a moderate disability, $41-60 \%$ severe disability, $61 \%$ to $80 \%$ for crippled and $81 \%$ to $100 \%$ 


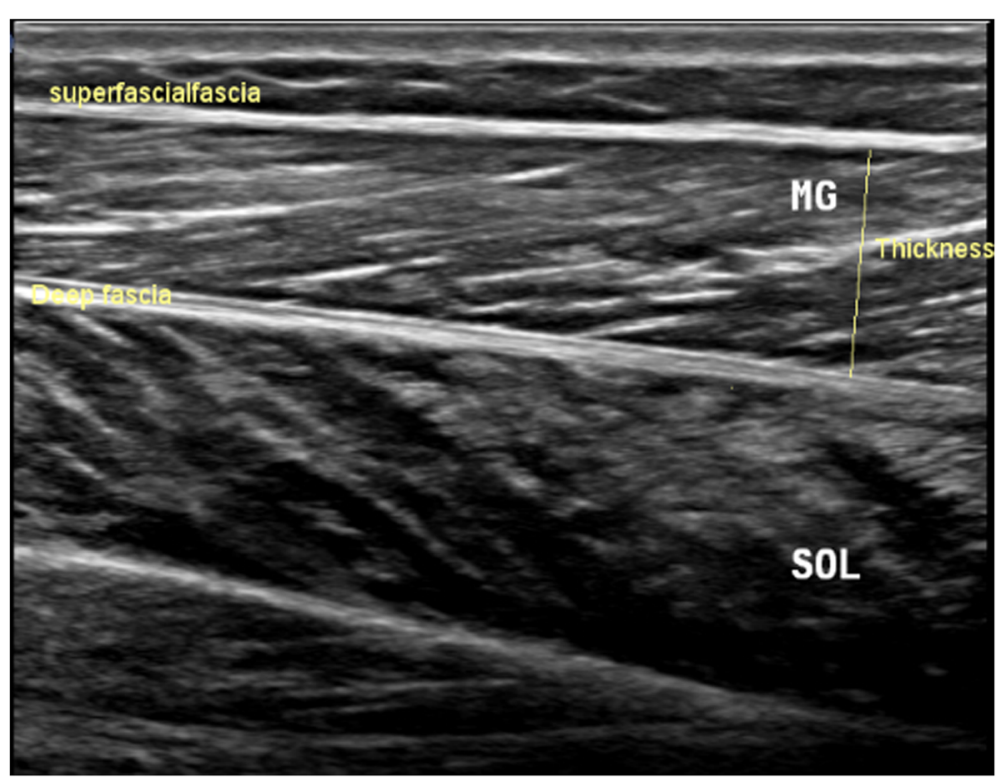

Fig. 1 Muscle thickness in the longitudinal scan, the distance between the superficial and deep fascia. In the the longitudinal scan, the US probe is placed parallel to the longitudinal axis of the target structure. MG: medial gastrocnemius muscle, SOL: soleus muscle

confirmed by associated disc bulging or herniation and nerve root compression between the vertebral level at L4-L5 and L5-S1 on MRI [1]. In addition, 15 asymptomatic individuals were recruited from the local population as the control group with no history of LBP during the preceding 6 months, or dysfunction in the low back, thoracic, pelvis, or lower extremities. The control group was matched with the patient group regarding age and gender. Patients completed the Persian version of the Oswestry Disability Index (ODI) [16], Tegner Activity Scale [17] and the Numerical Pain Rating Scale (NPRS) [18]. Tegner Activity Scale is a graduated list of activities of daily living, recreation, and competitive sports; and the score scales from 0 to 10 where 0 represents sick leave or disability pension, and 10 is participation in competitive sports [17]. Patients were excluded if they had tumor / malignancy or bony defects in the lumbar region on MRI, systemic myopathy/neuropathy, previous surgery in the region of assessment, or evidence of central sensitization in the mechanism of pain [1]. All participants signed a consent form and the study was approved by the Human Ethics Committee at Tehran University of Medical Sciences, Tehran, Iran. The data were collected from March to July 2017.

\section{Apparatus}

A diagnostic ultrasound imaging unit set in B-mode (Affinity 50 Philips-Netherland) with a linear-array probe with 7-12 MHz band frequency was used to record the images. The gain was set at $48 \%$ of the range, dynamic range was maintained at $93 \mathrm{~dB}$, and time compensation

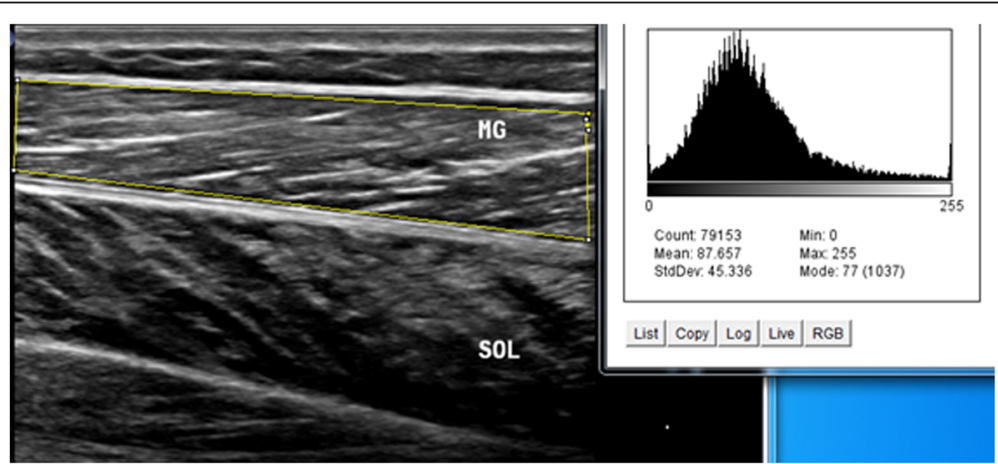

Fig. 2 Maximum region of interest $(\mathrm{ROI})$ with histogram echo intensity in the longitudinal scan. A maximum $\mathrm{ROI}$ was defined for each image to include as much of the muscle as possible, avoiding bone and surrounding fasciae. Histogram quantifies the greyscale of each pixel in arbitrary units. MG: medial gastrocnemius muscle, SOL: soleus muscle 


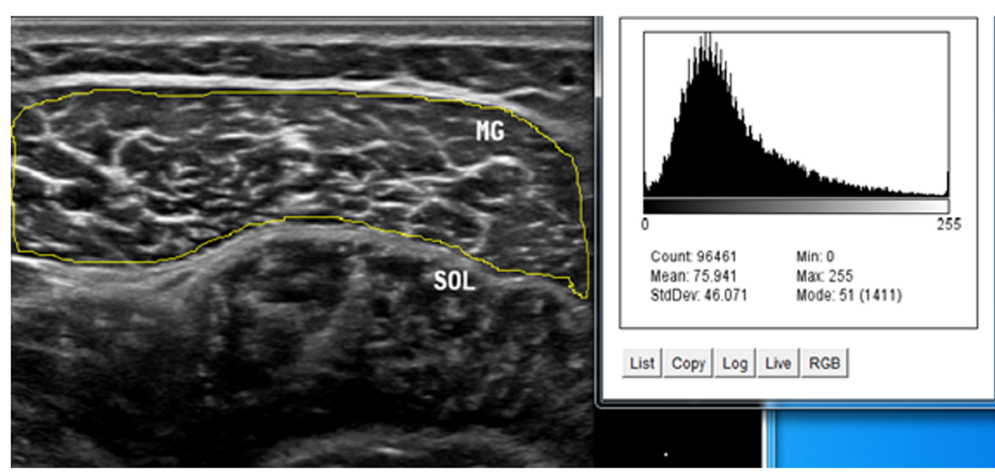

Fig. 3 Maximum region of interest with histogram echo intensity in the transverse scan. In the transverse scan, the US probe should be placed perpendicular to the structure of interest. MG: medial gastrocnemius muscle, SOL: soleus muscle

was kept at the same (neutral) position for all images' depths. The depth setting was adjusted for each muscle to visualize their superior and inferior margins. Images were recorded as JPEG files and stored on a computer for later processing. ImageJ software (Version 1.48v, National Institutes of Health, Bethesda, MD, USA) was utilized to calculate the muscle thickness and echo intensity as well as the sciatic nerve CSA and echo intensity.

\section{Data acquisition}

Intrarater within-day reliability of ultrasound measurements of contraction ratio and echo intensity was assessed in all the participants. First, the examiner performed all the measurements, and then, repeated the measurements after $60 \mathrm{~min}$ in a random order (condition names picked from a bowl) with the same procedure. To assess the intrarater reliability, three ultrasound images were acquired in the transverse and longitudinal views of four muscles, i.e. multifidus, biceps femoris, medial gastrocnemius, soleus. Between each scan, the probe was moved away from the nerve and muscle and then placed back again over the same area of the nerve and muscle for the next scan. All images were recorded at the end of expiration and they were captured from two sides within one session in the transverse and longitudinal views. Muscle thickness was measured as the largest distance between the superficial and deep fasciae, identified by their hyperechoic appearance in the longitudinal scan (Fig. 1). Two different ROIs were selected from the rest position to measure the echo intensity. First, maximum ROI was drawn for each scan to include as much of the muscle as possible, avoiding bone and surrounding fasciae in two scan views (Figs. 2 and 3). Second, to calculate max rectangular ROI, a rectangular ROI (as large as possible) was positioned over the inner region of muscle image in two scan views (Figs. 4 and 5). Echo intensity was then defined as the mean level of gray within the ROI in 8-bit resolution images (gray levels from 0 to 255 , where black $=0$ and white $=255)$ [7].

Direct and indirect methods were used to measure the CSA of the sciatic nerve. In the direct method, the inner border of the perineal echogenic rim that surrounds the hypoechoic sciatic nerve was traced (Fig. 6). The indirect method employed the formula for calculating an ellipsoid area (major diameter $\times$ minor diameter $\times 3.14 / 4$ ) (Fig. 7) [19]. The major diameter is defined as the longest line between two points of the nerve that passes through the center, whereas the minor diameter is the line through the

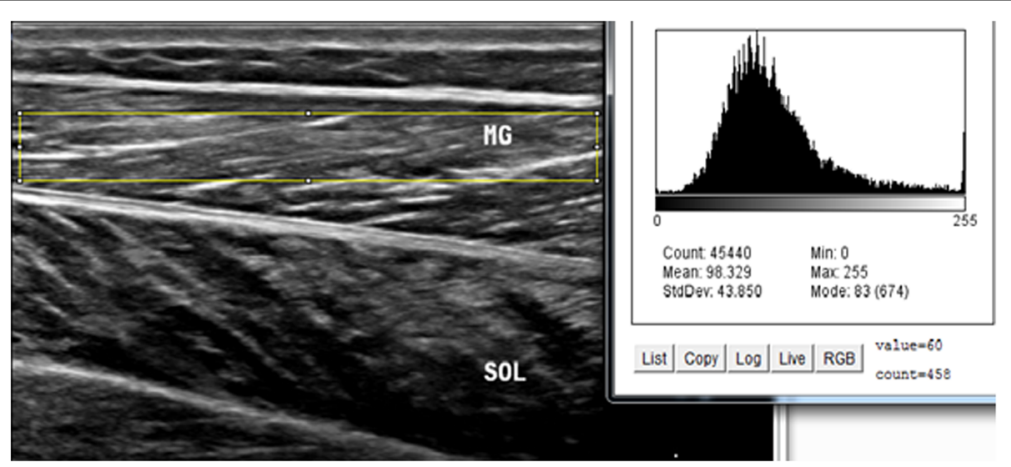

Fig. 4 Maximum rectangular region of interest with histogram echo intensity in the longitudinal scan. MG: medial gastrocnemius muscle, SOL: soleus muscle 


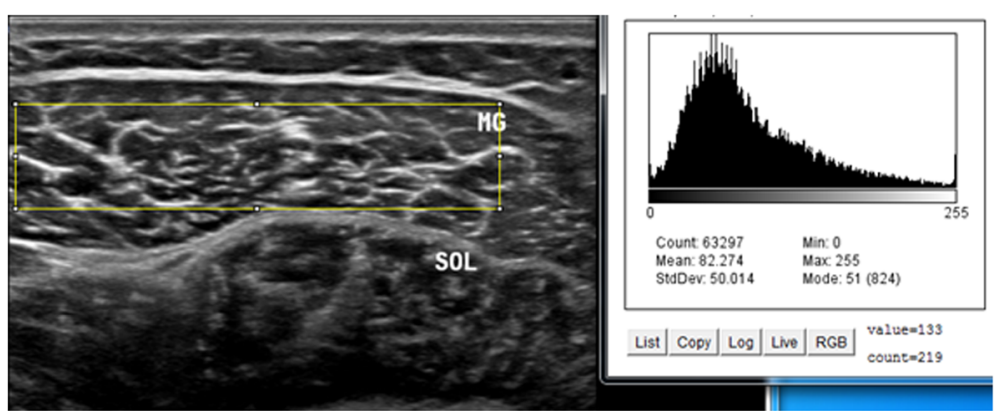

Fig. 5 Maximum rectangular region of interest (ROI) with histogram echo intensity in the transverse scan. Rectangular ROI was chosen in each scan to include as much of the muscle as possible without any bone or surrounding fascia. MG: medial gastrocnemius muscle, SOL: soleus muscle

center of the nerve perpendicular to the major diameter. All images were saved and exported for further analysis and the examiner was blind to the images' group allocation during processing the images by ImageJ software.

\section{Ultrasonography of the sciatic nerve and biceps femoris}

Ultrasonography of the sciatic nerve and biceps femoris muscle was performed at the level of lower $1 / 4$ on the posterior mid-thigh, along with a line from the ipsilateral iliac crest to the popliteal crease. Longitudinal scans were used to measure muscle thickness, and transverse scans provided a cross-sectional view of the biceps femoris and the sciatic nerve [20]. Participant performed an isometric prone hip extension with a straight knee (off the table to a height of $\sim 20 \mathrm{~cm}$ ) to activate the biceps femoris muscle.

\section{Ultrasonography of medial gastrocnemius and soleus}

The medial gastrocnemius and soleus muscles were captured at the point of lower $1 / 3$ of the tibial length from the midpoint of the medial malleolus to the popliteal crease [21]. Longitudinal scans were used to record the thickness

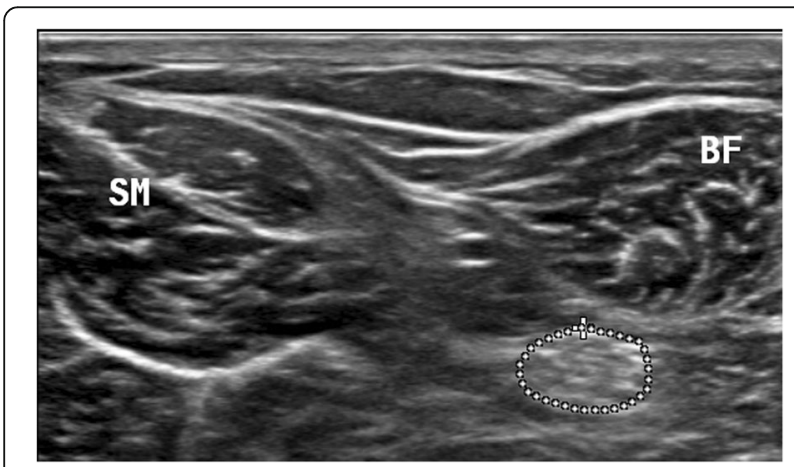

Fig. 6 Sciatic nerve with trace cross-sectional area. In the direct method, the inner border of the perineural echogenic rim that surrounds the hypoechoic sciatic nerve was traced, and measured nerve area by tracing along the hyperechoic epineurium, approximating inside of the epineurium. BF: biceps femoris muscle, SM: semimembranosus muscle of the soleus and medial gastrocnemius, while transverse scans provided only a cross-sectional view of the medial gastrosoleus. Participant rose on the toes $(5-\mathrm{cm}$ heel lift) while standing to activate the soleus and medial gastrocnemius.

\section{Ultrasonography of multifidus muscle}

The multifidus muscle was imaged at the L5 vertebral level of the lower back. To record longitudinal scans, the US probe was placed $\sim 2 \mathrm{~cm}$ lateral to the midline in a way having the spinal facet joints clearly in the image. To record transverse scans, the probe was spanned across the spinous processes to have bilateral cross-sectional views of the multifidus muscles. Participant laid prone and lifted the ipsilateral leg off the table to a height of $\sim 20 \mathrm{~cm}$ to activate the multifidus muscle [22].

\section{Statistical analysis}

Data were presented as means \pm standard deviations (SDs). All statistical analyses were performed with SPSS

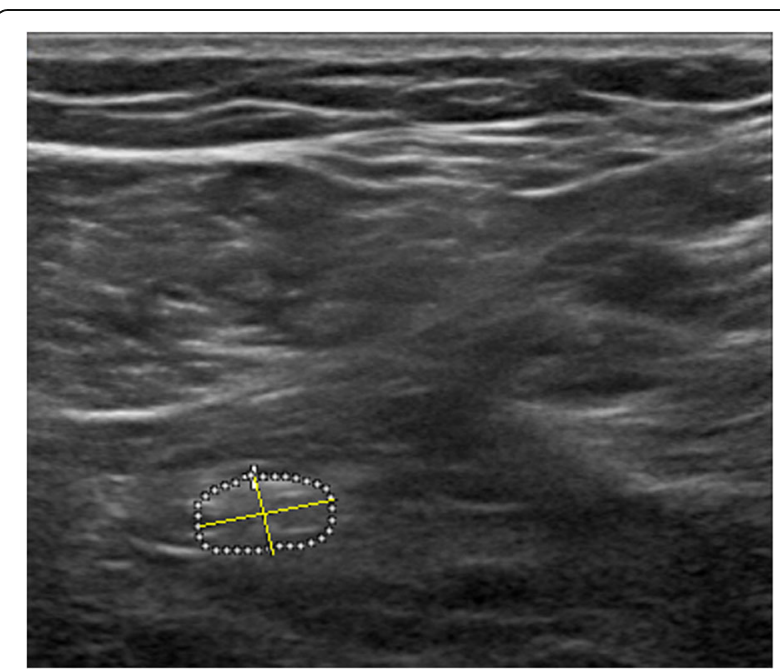

Fig. 7 Sciatic nerve with ellipse cross-sectional area. The major and minor diameters and the formula of an ellipsoid to calculate the area (major diameter $\times$ minor diameter $\times 3.14 / 4$ ) 
Table 2 Reliability of muscle contraction ratio in the controls (dominant and nondominant sides) and patients with sciatica (affected and unaffected sides)

\begin{tabular}{|c|c|c|c|c|c|}
\hline & Mean & SD & ICC & SEM & MDC \\
\hline \multicolumn{6}{|l|}{ Dominant } \\
\hline Multifidus & 1.24 & 0.19 & 0.82 & 0.08 & 0.22 \\
\hline Biceps Femoris & 1.17 & 0.14 & 0.91 & 0.04 & 0.11 \\
\hline Medial Gastrocnemius & 1.12 & 0.07 & 0.90 & 0.03 & 0.08 \\
\hline Soleus & 1.10 & 0.06 & 0.87 & 0.03 & 0.07 \\
\hline \multicolumn{6}{|l|}{ Nondominant } \\
\hline Multifidus & 1.25 & 0.17 & 0.84 & 0.06 & 0.18 \\
\hline Biceps Femoris & 1.20 & 0.13 & 0.90 & 0.03 & 0.14 \\
\hline Medial Gastrocnemius & 1.12 & 0.07 & 0.86 & 0.03 & 0.09 \\
\hline Soleus & 1.10 & 0.07 & 0.88 & 0.03 & 0.09 \\
\hline \multicolumn{6}{|l|}{ Affected } \\
\hline Multifidus & 0.87 & 0.40 & 0.85 & 0.05 & 0.16 \\
\hline Biceps Femoris & 1.13 & 0.25 & 0.89 & 0.08 & 0.22 \\
\hline Medial Gastrocnemius & 1.01 & 0.20 & 0.87 & 0.07 & 0.19 \\
\hline Soleus & 0.90 & 0.07 & 0.90 & 0.06 & 0.17 \\
\hline \multicolumn{6}{|l|}{ Unaffected } \\
\hline Multifidus & 1.20 & 0.14 & 0.89 & 0.04 & 0.13 \\
\hline Biceps Femoris & 1.27 & 0.16 & 0.90 & 0.04 & 0.13 \\
\hline Medial Gastrocnemius & 1.20 & 0.21 & 0.86 & 0.07 & 0.19 \\
\hline Soleus & 1.25 & 0.22 & 0.89 & 0.06 & 0.18 \\
\hline
\end{tabular}

SD Standard Deviation, ICC Intraclass correlation coefficient, SEM Standard Error of Measurement, MDC Minimal Detectable Change

statistical software version 24 (IBM Corporation, Chicago, IL, USA) software package. Intra-session reliability for the average of three measures in contraction ratio and echo intensity was assessed by the intraclass correlation coefficient (ICC 3, 1; method: alpha, two-way mixed, consistency). The ICCs are classified as follow: $<0.69$, poor correlation; $0.70-0.79$, fair correlation; $0.80-0.89$ good correlation; $0.90-1.00$ high correlation [23]. The standard error of measurement (SEM) and minimal detectable change (MDC) were also calculated to make a judgment about the degree that measurements vary for an individual. The SEM values indicate the precision of the measurement and were calculated based on the ICC and the SD of the mean of differences between the two measurements $[\mathrm{SEM}=\mathrm{SD} \sqrt{ } 1-\mathrm{ICC}]$. The MDC represents the smallest change in a score within an individual that can be considered as a real change above measurement error, which was determined using the formula: $[\mathrm{MDC}=$ $1.96 \times \sqrt{ } 2 \times \mathrm{SEM}][24]$.

\section{Results}

The demographic data for both patients and controls are summarized in Table 1 . Both groups had very similar age and BMI. The sciatica group had moderate to severe pain and disability. Reliability data of muscle contraction ratios are presented in Table 2. Within the patient group, the affected side had a lower contraction ratio with larger SD compared to the unaffected side. The reliability of the muscles was good to high (ICC $=0.82$ 0.91 ) in both groups. The SEM and MDC ranged from $0.03-0.08$ and $0.07-0.22$ in the controls, and 0.04-0.08 and $0.13-0.22$ in the sciatica group, respectively.

Table 3 reports the reliability and mean \pm SD of two methods (i.e., trace and ellipse) to measure the sciatic nerve CSA as well as echogenicity. In general, the trace method had higher ICC, and lower SEM and MDC compared to the ellipse method. Reliability of echogenicity ranged from 0.71 to 0.74 ; and 0.75 to 0.82 in the controls and patients, respectively.

Reliability and mean \pm SD of muscle echo intensity measures using max rectangular ROI with two different scans (transverse and longitudinal) are presented in Table 4. The longitudinal scan $(0.80-0.89)$ had slightly higher ICC values compared to the transverse scan $(0.75-0.87)$.

Table 5 shows the reliability and mean \pm SD of muscle echo intensity measures by maximum ROI with to scans (transverse and longitudinal). There was higher reliability with lower SEM and MDC for muscle echo intensity in the transverse scan relative to the longitudinal scan.

\section{Discussion}

In the current study, ultrasound imaging was used to evaluate the intrarater within-day reliability of nerve and muscle morphological features such as CSA, size, function, and

Table 3 Reliability of sciatic nerve cross-sectional area / echo intensity in the controls (dominant and nondominant sides) and patients with sciatica (affected and unaffected sides)

\begin{tabular}{|c|c|c|c|c|c|c|c|c|c|c|c|c|c|c|c|}
\hline & \multicolumn{10}{|c|}{ Cross-Sectional Area $\left(\mathrm{mm}^{2}\right)$} & \multicolumn{5}{|c|}{ Echogenicity (a.u.) } \\
\hline & \multicolumn{5}{|c|}{ Trace method } & \multicolumn{5}{|c|}{ Ellipse method } & & & & & \\
\hline & Mean & SD & ICC & SEM & $\mathrm{MDC}$ & Mean & SD & ICC & SEM & MDC & Mean & SD & ICC & SEM & MDC \\
\hline Dominant & 47.76 & 12.77 & 0.94 & 3.10 & 8.59 & 47.54 & 12.59 & 0.82 & 5.34 & 14.76 & 75.5 & 26.40 & 0.74 & 13.44 & 37.14 \\
\hline Nondominant & 48.01 & 12.3 & 0.97 & 2.14 & 5.94 & 47.98 & 12.51 & 0.88 & 4.33 & 11.96 & 74.98 & 21.07 & 0.71 & 11.24 & 31.07 \\
\hline Affected & 51.81 & 10.0 & 0.91 & 3.0 & 8.31 & 50.97 & 9.73 & 0.83 & 4.01 & 11.08 & 70.64 & 26.48 & 0.75 & 13.24 & 36.59 \\
\hline Unaffected & 45.54 & 9.11 & 0.90 & 2.88 & 7.97 & 45.11 & 8.98 & 0.81 & 3.91 & 10.81 & 71.67 & 37.28 & 0.82 & 15.81 & 43.70 \\
\hline
\end{tabular}

ICC Intraclass Correlation Coefficient, SEM Standard Error of Measurement, MDC Minimal Detectable Change, a.u. arbitrary units 
Table 4 Reliability of muscle echo-intensity measures for the maximum rectangular region of interest in the controls (dominant and nondominant sides) and patients with sciatica (affected and unaffected sides)

\begin{tabular}{|c|c|c|c|c|c|c|c|c|c|c|}
\hline & \multicolumn{5}{|c|}{ Transverse scan (a.u.) } & \multicolumn{5}{|c|}{ Longitudinal scan (a.u.) } \\
\hline & Mean & SD & $\mathrm{ICC}$ & SEM & $\mathrm{MDC}$ & Mean & SD & ICC & SEM & MDC \\
\hline \multicolumn{11}{|l|}{ Dominant } \\
\hline Multifidus & 90.53 & 18.27 & 0.81 & 7.95 & 21.99 & 91.83 & 23.20 & 0.83 & 9.56 & 26.42 \\
\hline Biceps Femoris & 67.89 & 21.26 & 0.76 & 10.40 & 28.74 & 59.08 & 16.86 & 0.80 & 7.54 & 20.84 \\
\hline Medial Gastrocnemius & 79.98 & 14 & 0.78 & 6.57 & 18.67 & 84.82 & 20 & 0.82 & 8.48 & 23.39 \\
\hline Soleus & 104.39 & 47.16 & 0.79 & 21.60 & 59.70 & 100.74 & 45.58 & 0.81 & 19.83 & 54.80 \\
\hline \multicolumn{11}{|l|}{ Nondominant } \\
\hline Multifidus & 98.13 & 20.1 & 0.75 & 10.05 & 27.79 & 89.75 & 17.31 & 0.80 & 7.74 & 21.39 \\
\hline Biceps Femoris & 81.90 & 23.23 & 0.77 & 11.13 & 30.75 & 78.74 & 21.48 & 0.82 & 9.11 & 25.17 \\
\hline Medial Gastrocnemius & 79.89 & 18.67 & 0.76 & 9.13 & 25.23 & 80.85 & 14.24 & 0.83 & 5.87 & 16.23 \\
\hline Soleus & 104.03 & 46.29 & 0.78 & 16.85 & 46.57 & 100.46 & 48.82 & 0.82 & 20.70 & 57.21 \\
\hline \multicolumn{11}{|l|}{ Affected } \\
\hline Multifidus & 99.41 & 41.53 & 0.82 & 17.61 & 48.69 & 98.89 & 41.1 & 0.84 & 16.44 & 45.43 \\
\hline Biceps Femoris & 73.71 & 37.82 & 0.85 & 14.64 & 40.45 & 80.21 & 42.72 & 0.80 & 19.10 & 52.79 \\
\hline Medial Gastrocnemius & 89.87 & 45.89 & 0.83 & 18.98 & 52.47 & 90.55 & 47.92 & 0.84 & 19.17 & 52.98 \\
\hline Soleus & 99.76 & 31.22 & 0.87 & 11.24 & 31.08 & 96.63 & 32.23 & 0.89 & 10.67 & 29.5 \\
\hline \multicolumn{11}{|l|}{ Unaffected } \\
\hline Multifidus & 98.74 & 42.28 & 0.82 & 17.93 & 49.57 & 99.76 & 43.87 & 0.84 & 17.55 & 48.50 \\
\hline Biceps Femoris & 72.11 & 39.23 & 0.80 & 17.54 & 48.49 & 74.10 & 38.03 & 0.83 & 15.67 & 43.32 \\
\hline Medial Gastrocnemius & 80.21 & 47.97 & 0.87 & 17.27 & 47.72 & 79.27 & 48.79 & 0.86 & 18.25 & 50.46 \\
\hline Soleus & 95.89 & 31.52 & 0.85 & 12.2 & 33.51 & 97.45 & 35.02 & 0.84 & 14.01 & 38.72 \\
\hline
\end{tabular}

ICC Intraclass correlation coefficient, SEM Standard Error of Measurement, MDC Minimal Detectable Change, arbitrary units (a.u)

quality of these structures in patients with LBP with unilateral radiculopathy (sciatica) and healthy controls. Acceptable level of reliability is a prerequisite for using ultrasound imaging as a valid measure of muscle activity to make decisions, especially in a clinical setting. In general, the results of this study suggest that there are good to high reliability of ultrasound measurements of the nerve and muscle contraction in healthy controls as well as in the patients with sciatica. These findings support previous studies reporting acceptable reliability for nerve CSA and muscle thickness / echo intensity by ultrasonography [7, 14, 25-27]. However, there are some factors which can affect reliability; for example, examiner's US experience, participants and the testing situation. Furthermore, several sources of error may affect the ultrasound measurements, such as positioning of the US probe, anatomical landmark detection and precision of marking the fascial bands [28].

\section{Reliability of contraction ratio}

To the best of our knowledge, this is the first study to evaluate test-retest reliability contraction ratio of the lower back and lower limb muscles affected by sciatica. Measuring muscle thickness using rehabilitative ultrasound imaging may be useful as an indicator of muscle function or neuromuscular motor control in the assessment of sciatica due to lumbar disc herniation. The contraction ratio had high reliability and may be used to assess the alterations of muscles morphology and function in patients with sciatica.

Multifidus activation by the abdominal hollowing and contralateral arm rising maneuver has been studied in individuals with and without LBP [29-32]. However, these studies have not used the prone hip extension maneuver to assess multifidus activation. Thus, this method of activation can be employed to contract multifidus in clinical or research settings.

The contraction ratio of measured muscles ranged from 0.87 to 1.27 with large variability. It may be due to the variability of motor control or muscle recruitment patterns in the participants. For example, reduced multifidus muscle contraction ratio on the affected side may result from pain or reflex inhibition due to the nerve root compression. It is also plausible that reduced soleus contraction ratio was a result of individual differences in preferential motor activation and/or co-contraction muscle synergy or antagonist into the muscle chain [33]. Reliability of architectural properties of the medial gastro-soleus had been widely studied previously [34]; however, there is no report regarding the reliability of the muscle contraction ratio of these muscles. 
Table 5 Reliability of muscle echo-intensity measures for the maximum region of interest in the controls (dominant and nondominant sides) and patients with low back pain (affected and unaffected sides)

\begin{tabular}{|c|c|c|c|c|c|c|c|c|c|c|}
\hline & \multicolumn{5}{|c|}{ Transverse scan (a.u.) } & \multicolumn{5}{|c|}{ Longitudinal scan (a.u.) } \\
\hline & $\overline{M e a n}$ & SD & ICC & SEM & $\overline{M D C}$ & Mean & SD & ICC & SEM & $\overline{M D C}$ \\
\hline \multicolumn{11}{|l|}{ Dominant } \\
\hline Multifidus & 64.51 & 15.1 & 0.91 & 4.53 & 12.51 & 66.56 & 23.17 & 0.80 & 10.36 & 28.64 \\
\hline Biceps Femoris & 69.28 & 26.81 & 0.93 & 7.08 & 19.58 & 59.07 & 18.56 & 0.82 & 7.86 & 21.73 \\
\hline Medial Gastrocnemius & 83.20 & 19.90 & 0.96 & 3.98 & 10.99 & 84.45 & 20.31 & 0.90 & 6.42 & 17.75 \\
\hline Soleus & 95.51 & 46.18 & 0.94 & 11.27 & 31.14 & 102.50 & 54.98 & 0.85 & 21.28 & 58.33 \\
\hline \multicolumn{11}{|l|}{ Nondominant } \\
\hline Multifidus & 45.29 & 29.37 & 0.92 & 8.29 & 22.91 & 66.50 & 18.37 & 0.89 & 6.07 & 16.80 \\
\hline Biceps Femoris & 81.98 & 20.81 & 0.94 & 5.08 & 14.05 & 86.32 & 30.77 & 0.87 & 11.08 & 30.64 \\
\hline Medial Gastrocnemius & 79.09 & 22.43 & 0.91 & 6.73 & 18.57 & 80.24 & 16.98 & 0.92 & 4.79 & 13.26 \\
\hline Soleus & 106.52 & 50.06 & 0.90 & 15.82 & 43.72 & 89.72 & 49.78 & 0.88 & 17.22 & 47.60 \\
\hline \multicolumn{11}{|l|}{ Affected } \\
\hline Multifidus & 108.19 & 42.37 & 0.94 & 10.34 & 28.58 & 99.08 & 36.48 & 0.82 & 15.47 & 42.77 \\
\hline Biceps Femoris & 73.32 & 39.14 & 0.92 & 11.04 & 30.52 & 78.82 & 40.37 & 0.84 & 16.15 & 44.63 \\
\hline Medial Gastrocnemius & 90.12 & 45.94 & 0.93 & 12.13 & 33.52 & 91.36 & 50.18 & 0.86 & 18.77 & 51.89 \\
\hline Soleus & 98.65 & 30.66 & 0.91 & 9.20 & 25.44 & 99.18 & 35.79 & 0.90 & 11.31 & 31.27 \\
\hline \multicolumn{11}{|l|}{ Unaffected } \\
\hline Multifidus & 101.90 & 35.88 & 0.92 & 10.12 & 27.98 & 106.13 & 44.59 & 0.81 & 19.40 & 53.61 \\
\hline Biceps Femoris & 81.12 & 38.33 & 0.92 & 10.81 & 29.89 & 83.48 & 35.77 & 0.83 & 14.74 & 40.75 \\
\hline Medial Gastrocnemius & 81.11 & 48.82 & 0.90 & 15.43 & 42.66 & 80.96 & 49.13 & 0.87 & 17.69 & 48.90 \\
\hline Soleus & 99.11 & 30.26 & 0.91 & 9.08 & 25.10 & 100.12 & 33.89 & 0.89 & 11.22 & 31.0 \\
\hline
\end{tabular}

ICC Intraclass correlation coefficient, SEM Standard Error of Measurement, MDC Minimal Detectable Change, a.u. arbitrary units

Ruas et al. (2017) also reported utilizing ultrasound imaging as a highly reliable method for the measuring of hamstrings muscle thickness [26]. Individuals may develop a protective motor strategy and adaptation (e.g., co-contraction of agonist-antagonist muscles, or increased activity of synergistic muscles) to pain provocation movement [35]. Due to the high reliability of the muscle contraction ratio, it can be considered as a potential outcome measure when assessing neuromotor function in clinical practice and in research. All the SEMs and the corresponding MDCs were low; thus making the contraction ratio a promising measure for future studies.

\section{Reliability of sciatic nerve morphological characteristics}

The reliability aspect of measuring CSA and echo intensity of the sciatic nerve with ultrasound in patients with LBP with unilateral radiculopathy (sciatica) has received little attention in the literature. This study showed that the sciatic nerve CSA had good to high test-retest reliability, while echo intensity had moderate reliability. The sciatic nerve was not always ellipsoid, therefore the tracing method was a slightly more reliable method than the ellipse method, which is comparable to the previous finding on the measurement of tibial nerve CSA [25]. Therefore, we suggest the direct method to determine the CSA of the sciatic nerve in this population. To our knowledge, little is known about the magnitude of SEM or MDC of the sciatic nerve in these patients. The SEM and MDC of CSA by tracing method were lower than ellipsoid in the controls. A similar trend was observed in the patient group. In line with our findings, two previous studies had reported acceptable reliability when measuring the sciatic nerve CSA [14, 27]. We found moderate reliability for the nerve echo intensity. However, no study has investigated the reliability of the echo intensity of the sciatic nerve in this population. Variability in the MDC measurements of sciatic nerve echo intensity may be due to the echogenic properties of surrounding tissues [13].

\section{Muscle echo intensity}

Muscle echo intensity values might offer important insight into the muscle changes caused by disease / pathological disorders [36], especially because it is more objective and possibly more reliable than a simple visual assessment of ultrasound images [37]. We observed moderate reliability of echo intensity using max rectangular ROI in the transverse scan. This finding is in 
line with Varanoske et al. (2017), who stated echo intensity may be heterogeneous when examining a portion of individual muscles in the transverse plane [38].

One of our aims was to determine the effect of ROI size on the reliability of ultrasound muscle echo intensity measures. Caresio et al. (2015) reported ICCs of 0.54-0.86 for within-session tibialis anterior and gastrocnemius muscle echo intensity depending on ROI size, with larger ROIs being associated with higher reliability [7]. In our study, using max rectangular ROI displayed lower SEM values for the longitudinal scan but larger SEM values for the transverse scan. Our results also showed that echo intensity reliability was generally lower than the contraction ratio nearly in all the assessed muscles. Lower echo intensity reliability can be due to variability in the US probe placement and potential image-to-image differences in background brightness, which may in return affect the ultrasound absorption and reflection of echo signals [39].

\section{Limitations}

One of the limitations of this study was calculating only intrarater within-day reliability. However, we decided to measure within-day reliability because changes in the hydration level, posture and muscle relaxation between the sessions may affect between-day reliability. Second, this study was performed in the middle-aged population with 1-3 activity level, which limits generalizability to other age groups with higher activity level (e.g., athletes). Another potential limitation was having only one rater, so consistency across multiple raters is unknown.

\section{Conclusions}

This study suggests moderate to high intrarater within-day reliability in muscle contraction ratio and muscle and sciatic nerve CSA and echogenicity in patients with LBP with unilateral radiculopathy and healthy controls. The reliability of echo intensity measurements is sensitive to ROI size; i.e., the maximum ROI in the transverse scan had the highest reliability. These findings can contribute to support the use of ultrasound for reliable evaluation of neuromuscular morphology and particularly to assess the small changes in both nerve and muscle structures in LBP with unilateral radiculopathy.

\section{Abbreviations}

CSA: Cross-sectional area; ICC: Intraclass correlation coefficient; LBP: Low back pain; MDC: Minimal detectable change; ROI: Region of interest; SD: Standard deviations; SEM: Standard error of measurement

\section{Acknowledgments}

The authors would like to acknowledge the generous assistance of Mrs. Ahmadi staff of Sonography Unit at Imam Khomeini Hospital, Tehran University of Medical Sciences. The authors thank Mrs. Rhona Gray for proofreading the mansucript.

\section{Funding}

This research was funded by Tehran University of Medical Science \& Health Services, International Campus (TUMS, IC-TUMS).

Availability of data and materials

The datasets generated and analyzed during the current study are available from the corresponding author upon request.

\section{Authors' contributions}

$\mathrm{HS}, \mathrm{MRH}, \mathrm{GO}$ and $\mathrm{HB}$ were responsible for the study design. HS and NAZ collected and prepared the data. HS, OR and SJ analyzed the data. HS and OR were responsible for writing and redrafting the manuscript. All authors read and approved the final manuscript.

\section{Ethics approval and consent to participate}

All participants signed a consent form and the study was approved by the Human Ethics Committee at Tehran University of Medical Sciences, Tehran, Iran.

\section{Consent for publication}

Written informed consent was obtained from the participants for publication of their individual details and accompanying images in this manuscript. The consent form is held by the first author and is available for review by the Editor-in-Chief.

\section{Competing interests}

The authors declare that they have no competing interests.

\section{Publisher's Note}

Springer Nature remains neutral with regard to jurisdictional claims in published maps and institutional affiliations.

\section{Author details}

'Department of Physiotherapy, School of Rehabilitation, International Campus, Tehran University of Medical Sciences (IC-TUMS), Tehran, Iran. ${ }^{2}$ Department of Neurology, Shahid Mohammadi Hospital, Hormozgan University of Medical Sciences, Bandar Abbas, Iran. ${ }^{3}$ Department of Physiotherapy, School of Rehabilitation, Tehran University of Medical Sciences, International Campus (TUMS, IC-TUMS), Brain and Spinal Injury Research Center (BASIR), Tehran, Iran. ${ }^{4}$ Radiology, Advanced Diagnostic and Interventional Radiology (ADIR) research center, Tehran University of Medical Sciences, Tehran, Iran. ${ }^{5}$ Department of Physiotherapy, School of Rehabilitation, Tehran University of Medical Sciences, (TUMS), Tehran, Iran. ${ }^{6}$ Biostatistics, Department of Physiotherapy, School of Rehabilitation, Tehran University of Medical Sciences, (TUMS), Tehran, Iran. ${ }^{7}$ Department of Mental Health, Faculty of Medicine and Health Sciences, Norwegian University of

Science and Technology (NTNU), Trondheim, Norway.

Received: 24 March 2018 Accepted: 3 September 2018

Published online: 06 November 2018

\section{References}

1. Nijs J, Apeldoorn A, Hallegraeff H, Clark J, Smeets R, Malfliet A, et al. Low back pain: guidelines for the clinical classification of predominant neuropathic, nociceptive, or central sensitization pain. Pain Physician. 2015;18(3):E333-46.

2. Checchia GA, Letizia Mauro G, Morico G, Oriente A, Lisi C, Polimeni V, et al. Observational multicentric study on chronic sciatic pain: clinical data from 44 Italian centers. Eur Rev Med Pharmacol Sci. 2017;21(7):1653-64.

3. Hobson-Webb LD, Cartwright MS. Advancing neuromuscular ultrasound through research: finding common sound. Muscle Nerve. 2017;56(3):375-8.

4. Vlychou M, Teh J. Ultrasound of muscle. Curr Probl Diagn Radiol. 2008;37(5): 219-30

5. Mayans D, Cartwright MS, Walker FO. Neuromuscular ultrasonography: quantifying muscle and nerve measurements. Phys Med Rehabil Clin N Am. 2012;23(1):133-48. xii

6. Arab AM, Rasouli O, Amiri M, Tahan N. Reliability of ultrasound measurement of automatic activity of the abdominal muscle in participants with and without chronic low back pain. Chiropr Man Therap. 2013;21(1):37.

7. Caresio C, Molinari F, Emanuel G, Minetto MA. Muscle echo intensity: reliability and conditioning factors. Clin Physiol Funct Imaging. 2015;35(5): 393-403. 
8. Santos R, Armada-da-Silva PAS. Reproducibility of ultrasound-derived muscle thickness and echo-intensity for the entire quadriceps femoris muscle. Radiography (Lond). 2017;23(3):e51-61.

9. O'Brien TG, Cazares Gonzalez ML, Ghosh PS, Mandrekar J, Boon AJ. Reliability of a novel ultrasound system for gray-scale analysis of muscle. Muscle Nerve. 2017:56(3):408-12.

10. Boom J, Visser LH. Quantitative assessment of nerve echogenicity: comparison of methods for evaluating nerve echogenicity in ulnar neuropathy at the elbow. Clin Neurophysiol. 2012;123(7):1446-53.

11. Fink A, Teggeler M, Schmitz M, Janssen J, Pisters M. Reproducibility of ultrasonographic measurements of the ulnar nerve at the cubital tunnel. Ultrasound Med Biol. 2017;43(2):439-44.

12. Stolz LA, Acuna JG, Gaskin K, Murphy AM, Friedman L, Stears-Ellis S, et al. Echogenicity and ultrasound visibility of peripheral nerves of the upper extremity. Med Ultrason. 2018;20(2):199-204.

13. Boehm J, Scheidl E, Bereczki D, Schelle T, Aranyi Z. High-resolution ultrasonography of peripheral nerves: measurements on 14 nerve segments in 56 healthy subjects and reliability assessments. Ultraschall Med. 2014; 35(5):459-67.

14. Cartwright MS, Demar S, Griffin LP, Balakrishnan N, Harris JM, Walker FO. Validity and reliability of nerve and muscle ultrasound. Muscle Nerve. 2013; 47(4):515-21.

15. Kerasnoudis A, Tsivgoulis G. Nerve ultrasound in peripheral neuropathies: a review. J Neuroimaging. 2015;25(4):528-38.

16. Mousavi SJ, Parnianpour M, Mehdian H, Montazeri A, Mobini B. The Oswestry disability index, the Roland-Morris disability questionnaire, and the Quebec back pain disability scale: translation and validation studies of the Iranian versions. Spine (Phila Pa 1976). 2006;31(14):E454-9.

17. Tegner $Y$, Lysholm J. Rating systems in the evaluation of knee ligament injuries. Clin Orthop Relat Res. 1985198:43-9.

18. Stratford PW, Spadoni G. The reliability, consistency, and clinical application of a numeric pain rating scale. Physiother Can. 2001;53(2):88-91.

19. Yesildag A, Kutluhan S, Sengul N, Koyuncuoglu HR, Oyar O, Guler K, et al. The role of ultrasonographic measurements of the median nerve in the diagnosis of carpal tunnel syndrome. Clin Radiol. 2004;59(10):910-5.

20. Frost LR, Brown SH. Neuromuscular ultrasound imaging in low back pain patients with radiculopathy. Man Ther. 2016;21(83-8.

21. Cho KH, Lee HJ, Lee WH. Reliability of rehabilitative ultrasound imaging for the medial gastrocnemius muscle in poststroke patients. Clin Physiol Funct Imaging. 2014;34(1):26-31.

22. Bruno PA, Millar DP, Goertzen DA. Inter-rater agreement, sensitivity, and specificity of the prone hip extension test and active straight leg raise test. Chiropractic \& manual therapies. 2014;22(1):23.

23. Blesh T. Measurement in physical education. New York, NY: The Ronald Press Company; 1974.

24. Weir JP. Quantifying test-retest reliability using the intraclass correlation coefficient and the SEM. J Strength Cond Res. 2005;19(1):231-40.

25. Alshami AM, Cairns CW, Wylie BK, Souvlis T, Coppieters MW. Reliability and size of the measurement error when determining the cross-sectional area of the tibial nerve at the tarsal tunnel with ultrasonography. Ultrasound Med Biol. 2009;35(7):1098-102.

26. Ruas CV, Pinto RS, Lima CD, Costa PB, Brown LE. Test-retest reliability of muscle thickness, echo-intensity and cross sectional area of quadriceps and hamstrings muscle groups using B-mode ultrasound. International Journal of Kinesiology and Sports Science. 2017;5(1):35-41.

27. Tagliafico A, Cadoni A, Fisci E, Bignotti B, Padua L, Martinoli C. Reliability of side-to-side ultrasound cross-sectional area measurements of lower extremity nerves in healthy subjects. Muscle Nerve. 2012;46(5):717-22.

28. Costa LO, Maher CG, Latimer J, Hodges PW, Shirley D. An investigation of the reproducibility of ultrasound measures of abdominal muscle activation in patients with chronic non-specific low back pain. Eur Spine J. 2009;18(7):1059-65.

29. Djordjevic O, Konstantinovic L, Miljkovic N, Bijelic G. Relationship between electromyographic signal amplitude and thickness change of the trunk muscles in patients with and without low back pain. Clin J Pain. 2015;31(10):893-902.

30. Hides JA, Miokovic T, Belavy DL, Stanton WR, Richardson CA. Ultrasound imaging assessment of abdominal muscle function during drawing-in of the abdominal wall: an intrarater reliability study. J Orthop Sports Phys Ther. 2007;37(8):480-6.

31. Kiesel KB, Uhl TL, Underwood FB, Rodd DW, Nitz AJ. Measurement of lumbar multifidus muscle contraction with rehabilitative ultrasound imaging. Man Ther. 2007;12(2):161-6.
32. Koppenhaver SL, Hebert JJ, Fritz JM, Parent EC, Teyhen DS, Magel JS. Reliability of rehabilitative ultrasound imaging of the transversus abdominis and lumbar multifidus muscles. Arch Phys Med Rehabil. 2009;90(1):87-94.

33. Frost $L R$, Brown SH. Muscle activation timing and balance response in chronic lower back pain patients with associated radiculopathy. Clin Biomech (Bristol, Avon). 2016;32(124-30.

34. Kwah LK, Pinto RZ, Diong J, Herbert RD. Reliability and validity of ultrasound measurements of muscle fascicle length and pennation in humans: a systematic review. J Appl Physiol (1985). 2013;114(6):761-9.

35. Hodges PW. Pain and motor control: from the laboratory to rehabilitation. J Electromyogr Kinesiol. 2011;21(2):220-8.

36. Arts IM, Pillen S, Schelhaas HJ, Overeem S, Zwarts MJ. Normal values for quantitative muscle ultrasonography in adults. Muscle Nerve. 2010;41(1):32-41.

37. Pillen S, van Keimpema M, Nievelstein RA, Verrips A, van KruijsbergenRaijmann W, Zwarts MJ. Skeletal muscle ultrasonography: Visual versus quantitative evaluation. Ultrasound Med Biol. 2006;32(9):1315-21.

38. Varanoske AN, Fukuda DH, Boone CH, Beyer KS, Stout JR, Hoffman JR. Homogeneity of echo intensity values in transverse ultrasound images. Muscle Nerve. 2017;56(1):93-8.

39. Young HJ, Jenkins NT, Zhao Q, McCully KK. Measurement of intramuscular fat by muscle echo intensity. Muscle Nerve. 2015;52(6):963-71.

Ready to submit your research? Choose BMC and benefit from:

- fast, convenient online submission

- thorough peer review by experienced researchers in your field

- rapid publication on acceptance

- support for research data, including large and complex data types

- gold Open Access which fosters wider collaboration and increased citations

- maximum visibility for your research: over $100 \mathrm{M}$ website views per year

At $\mathrm{BMC}$, research is always in progress.

Learn more biomedcentral.com/submissions 